\title{
The Unfolded Protein Response and its potential role in Huntington's disease
}

\author{
Ravi Kiran Reddy Kalathur, Kameshwar Ayasolla and Matthias E. Futschik* \\ Center for Molecular and Structural Biomedicine, University of Algarve, Faro, Portugal \\ *Correspondence to mfutschik@ualg.pt
}

\begin{abstract}
Huntington's disease (HD) is a progressive, neurodegenerative disease with fatal outcome. Although the diseasecausing gene (huntingtin) has been known for some time, the exact cause of neuronal cell death is still unknown. One potential mechanism contributing to the massive loss of neurons in the brain of HD patients might be the unfolded protein response (UPR), which is activated by accumulation of misfolded proteins in the endoplasmatic reticulum (ER). As an adaptive response to counter-balance accumulation of un- or misfolded proteins, the UPR upregulates transcription of chaperones, temporarily attenuates new translation, and activates protein degradation via the proteasome. However, it is known that persistent ER stress and activated UPR can cause cell death by triggering of apoptosis. Nevertheless, the evidence linking UPR with HD progression remains inconclusive. Here, we present first analyses of UPR activation during HD based on available expression data. To elucidate the potential role of UPR as a disease-relevant process, we examine its connection to cell death and inflammatory processes. Due to the complexity of these molecular mechanisms, a systems biology approach was pursued.
\end{abstract}

HD is an autosomal-dominant neurodegenerative disorder. Its symptoms are loss of motor control, cognitive decline, and behavioral abnormalities. For most cases, the disease onset is between the age of 35 and 50 . The outcome is fatal with life expectancy after disease onset of around 20 years only. The treatment of HD has remained symptomatic, as no cure exists to date.

The cause of HD is a mutation in a single gene called huntingtin. In HD patients, a expansion of the CAG repeat in exon 1 of huntingtin has been identified [1]. It results in a long stretch polyglutamine close to the $\mathrm{N}$-terminus of the Huntingtin protein (Htt) which is involved in multiple molecular functions. Although the molecular cause has been known now for almost 20 years, the exact mechanisms leading to the observed massive cell death of neurons in the caudate nucleus of HD patients still need to be determined. A variety of processes such excitotoxicity, protein aggregation and transcriptional dys-regulation have been suggested to be involved in neurodegeneration in HD. Only recently, studies have indicated that the ER stress and UPR might be linked to neurodegenerative diseases.

\section{ER stress and UPR}

The endoplasmic reticulum (ER) is a crucial organelle for correct folding and modification of numerous proteins. Accumulation of unfolded or misfolded protein causes a stress signal in the ER [2]. For HD, experimental evidence suggests that cytosolic mHtt fragments strongly impair endoplasmic reticulum (ER)-associated protein degradation (ERAD), as mHtt entraps ERAD proteins [3]. As the protein degradation is impaired, misfolded proteins might accumulate in the ER inferring with its correct functing. To ensure fidelity of protein folding, several transcriptional and translational mechanisms are activated during the
Unfolded Protein Response (UPR) which senses when the ER exceeded its capacity for correct folding. UPR stress sensors Inositol-requiring protein-1(IRE1), activating transcription factor 6 (ATF-6) and PKR-like ER kinase (PERK are subsequently activated. As an adaptive response to counter-balance accumulation of un- or misfolded proteins, the UPR upregulates transcription of chaperones, temporarily attenuates new translation, and activates protein degradation via the proteasome [4]. The main function of the UPR is re-establishing homeostasis by increasing the folding capacity.

Though the primary role of UPR is up-regulation of chaperones, persistent ER stress can mediate toxicity and eventually lead to apoptosis through activation of JNK, ASK1 and caspase-12. Remarkably, recent studies have also suggested that ER stress and activated UPR are interconnected with inflammatory processes [5]. Notably, secreted inflammatory cytokines and activated microglia cells could possibly lead to axonal damage and extensive neuronal cell death in HD pathology [6-9]. A scheme of the different mechanisms triggered by ER stress in the context of HD can be found in figure 1 .

As a potentially important process in HD progression, we examined therefore the activation of UPR during HD, and cross-talk between UPR and neurodegeneration in HD. Due to the complexity of these processes, we have started to develop explicit models for the interconnections between ER stress and apoptosis or neuroinflammation. Based on these models, we can suggest defined set of follow-up experiments to further clarify the role of UPR signaling related to pathophysiology in HD. As lowering of stress might prove beneficial, our objectives are a) to investigate the participation of molecular signaling networks and b) to identify novel drug targets. 


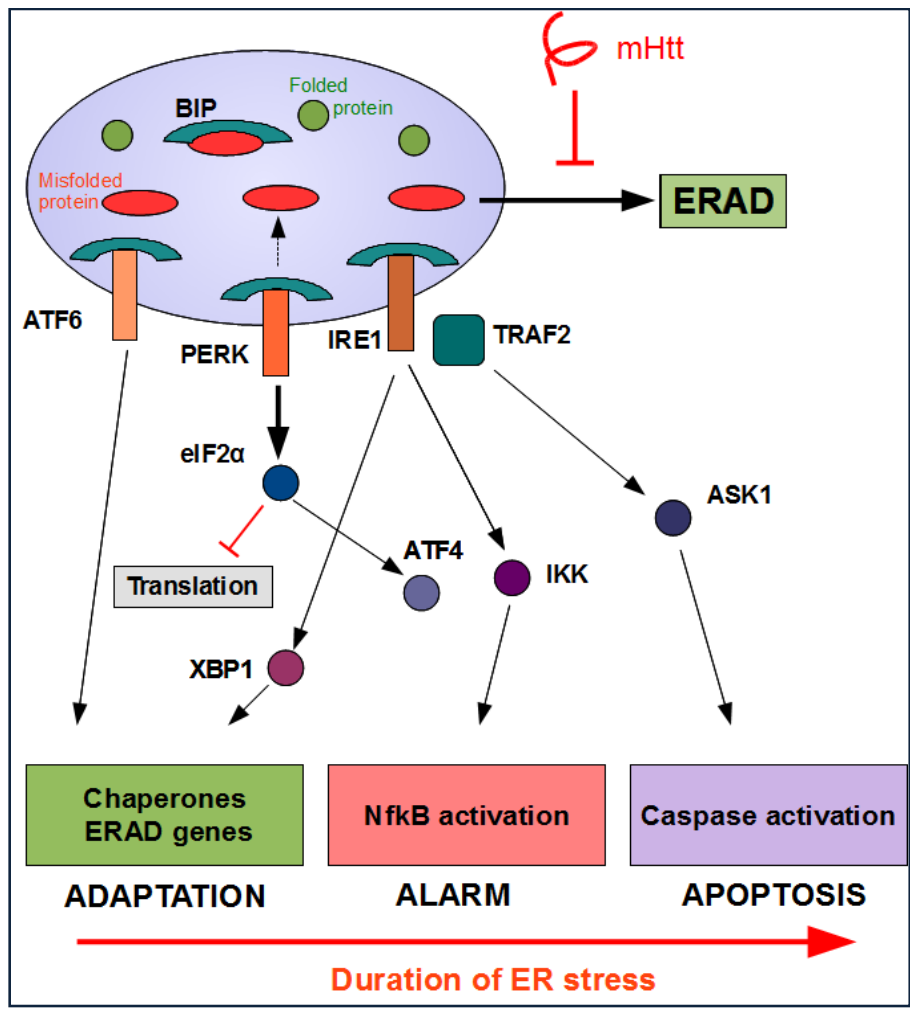

Figure 1. HD and possible UPR-associated mechanisms during disease progression. Mutant Huntingtin can interfere with ERassociated degradation (ERAD) resulting in accumulation of misfolded protein in the ER and thus in the activation of the UPR [3]. As an adaptive response, the UPR up-regulates transcription of chaperones, temporarily attenuates new translation, and activates protein degradation via the proteasome. Persistent levels of ER stress, however, may trigger inflammatory pathways as well as caspase activation and may ultimately lead to the induction of neuronal cell death.

\section{The UPR signaling network}

To determine possible implications of the UPR in HD, we seek to assess its activation using a computational approach and the evaluation of existing data. As a first step, the UPR signaling pathway has been reconstructed based on a literature review. The main branches are via ATF6, PERK and IRE1.

Although several main components have been well studied, it is less clear how alternative branches of the UPR are coordinated. Therefore, we have complemented the canonical UPR pathway with additional protein interaction data to represent its embedding into the cellular context and to identify potential modifiers. To this end, we have utilized the Unified Human Interactome (UniHI) database (www.unihi.org) to obtain interaction partners for EIF2AK3, ATF6, ERN1 and ATF4. The retrieved set of interactions partners defined a core set of proteins for the UPR (table 1). Figure 2 displays the derived UPR network consisting of 26 proteins.

\section{Potential activation of UPR in HD}

Since changes in gene transcription are main effects of UPR activation and published microarray data is available for different stages of HD in humans as well as for HD model, we used 3 different gene expression datasets in this study. Two datasets included expression for HD mouse models [10,11] and one dataset included expression for human HD brains and controls [12]. All data was obtained from the NCBI GEO database
(GSE10263, GSE3621 and GSE 3790) and analyzed using the R/Bioconductor platform.

To identify an expression signature for potential activation of the UPR in HD, we collected a set of known UPR target genes through literature review. To check whether these genes have ER stress elements (ERSE:CCAAT(N9)CCACG) and UPR elements (UPRE:CNTNKNNG)(GRAMYKGR)(ATACGGAG)) in their promoter sequences, we additionally obtained promoter sequences $(+5000$ to $-1000 \mathrm{bp})$ from TRANSFAC database and search them for ERSE and UPRE sequences.

Our initial analysis indicates that indeed the ER stress and UPR related genes are regulated during early stages of the HD and most of genes involved in UPR posses UPRE element (Figure 2) in their promoter and these elements play crucial role in the regulating these genes, although this expression signature needs further study.

\section{Cross-talk between UPR and apoptosis}

The set of genes involved in apoptosis was defined by the corresponding KEGG pathway. In order to identify cross-talk between UPR and apoptosis, we have distinguished genes that are either directly (core UPR and core apoptosis genes) or indirectly (extended UPR and extended apoptosis genes) involved in both UPR and apoptosis. Finally, we ended up with 26 core UPR 


\begin{tabular}{|c|c|c|c|c|c|}
\hline \multirow[t]{2}{*}{ Gene symbol } & \multirow[t]{2}{*}{ Gene Name } & \multicolumn{4}{|c|}{ Binding partner } \\
\hline & & EIF2AK3 & ATF6 & ERN1 & ATF4 \\
\hline ATF6 & activating transcription factor 6 & & & & \\
\hline ATF6B & activating transcription factor 6 beta & & $\bullet$ & & \\
\hline BAK1 & BCL2-antagonist/killer 1 & & & $\bullet$ & \\
\hline BAX & BCL2-associated X protein & & & $\bullet$ & \\
\hline BCL6 & B-cell CLL/lymphoma 6 (zinc finger protein 51) & $\bullet$ & & & \\
\hline COPS5 & COP9 constitutive photomorphogenic homolog subunit 5 & & & $\bullet$ & \\
\hline CREB3L3 & cAMP responsive element binding protein 3 like 3 & & $\bullet$ & & \\
\hline DNAJC3 & DnaJ (Hsp40) homolog, subfamily C, member 3 & $\bullet$ & & & \\
\hline EIF2AK3 & Eukaryotic translation initiation factor 2-alpha kinase 3 & & & & $\bullet$ \\
\hline EIF2S1 & eukaryotic translation initiation factor 2 , subunit 1 alpha, $35 \mathrm{kDa}$ & $\bullet$ & & & $\bullet$ \\
\hline ERN1 & endoplasmic reticulum to nucleus signalling 1 & & & & \\
\hline GTF2I & General transcription factor II-I & & - & & \\
\hline GTPBP2 & GTP binding protein 2 & & $\bullet$ & & \\
\hline HSP90AA1 & Heat shock protein HSP 90-alpha (HSP 86) & $\bullet$ & & $\bullet$ & \\
\hline HSP90B1 & heat shock protein 90kDa beta (Grp94), member 1 & $\bullet$ & & & \\
\hline HSPA5 & heat shock 70kDa protein 5 (glucose-regulated protein, 78kDa) & $\bullet$ & & $\bullet$ & \\
\hline IKBKB & Inhibitor of nuclear factor kappa B kinase beta subunit & $\bullet$ & & & \\
\hline MAP3K5 & mitogen-activated protein kinase kinase kinase 5 & & & $\bullet$ & \\
\hline NFE2L2 & nuclear factor (erythroid-derived 2)-like 2 & $\bullet$ & & & \\
\hline NFYC & nuclear transcription factor Y, gamma & & $\bullet$ & & \\
\hline PSEN1 & presenilin 1 & & & $\bullet$ & \\
\hline SRF & serum response factor & & - & & \\
\hline TAOK3 & TAO kinase 3 & & & $\bullet$ & \\
\hline TRAF2 & TNF receptor-associated factor 2 & & & $\bullet$ & \\
\hline XBP1 & X-box binding protein 1 & & $\bullet$ & & \\
\hline YY1 & YY1 transcription factor & & $\bullet$ & & \\
\hline
\end{tabular}

Table 1: List of proteins in the UPR signaling network. Physical protein-protein interactions with EIF2AK3, ATF6, ERN1 and ATF4 as derived from the UniHI database are noted.

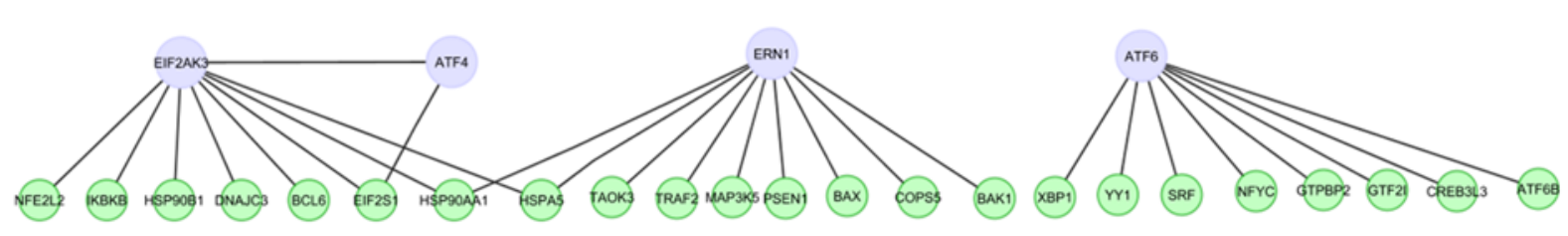

Figure 2: Protein interaction network for the UPR. Gray nodes represent the central UPR proteins EIF2AK3,

genes, 62 core apoptosis genes, 682 genes in extended UPR and 1414 genes in extended apoptosis (Figure 4).

Genes common to UPR and apoptosis are primary candidates constituting a cross-talk between both processes. Examination of the interaction networks indeed revealed that three proteins are associated with UPR as well as apoptosis (Figure 5):

- $\quad$ TRAF2 (TNF receptor-associated factor 2): ER stress-induced neuronal death can be activated by binding of ASK1 to the complex of IRE1 and TRAF2 [13].
- $\quad$ IKBKB (IkB-kinase beta): Phosphorylation of IkB proteins by IKBKB leads to their ubiquitination and destruction, thereby allowing activation of the NF-kappa-B complex.

- $\quad$ BAX (BCL2-associated X protein): This protein forms a heterodimer with BCL2, and acts as an apoptotic activator.

For a more complete representation of UPR-induced apoptosis, we intend to construct a dynamic network representation. Such model may also serve as rationale basis for experimental studies. 

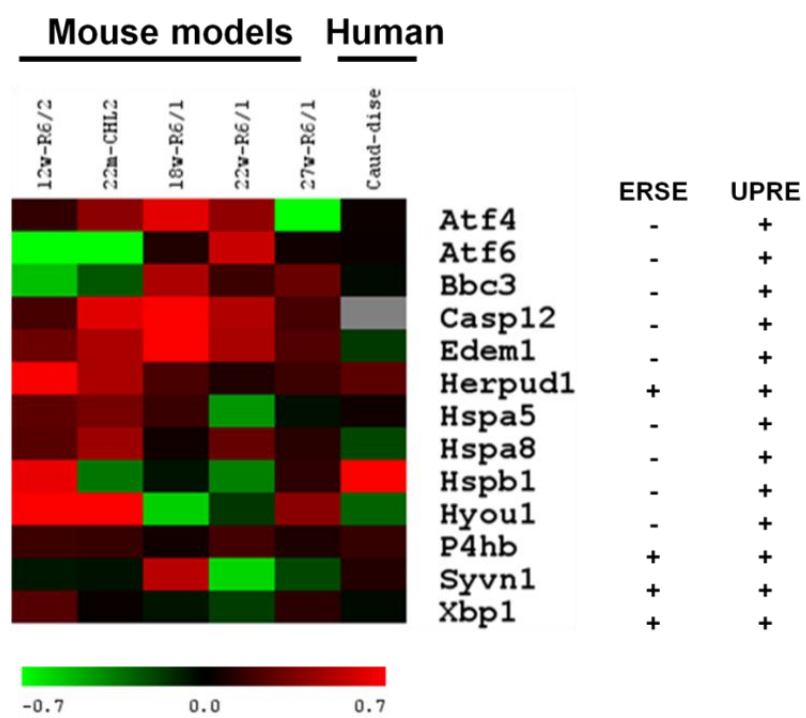

Figure 3: Expression of UPR-associated genes in HD mouse models (1-5 columns from left) and human HD patients (6th column). Up-regulation is represented by shades of red, down-regulation is represented by shades of green. Additionally, the presence of ERSE and UPRE in the promotor regions is indicated: + '= present; ' $'$ ' $=$ absent.

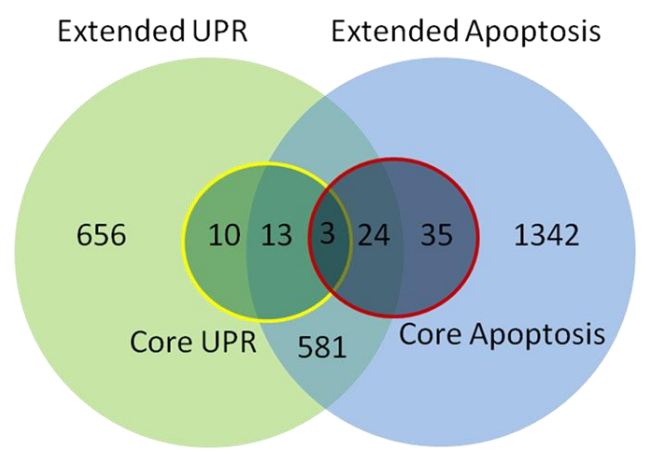

Figure 4: Venn diagram for core and extended UPR and apoptosis network. Extended UPR and apoptosis includes proteins that physically interact with core proteins. Interactions were derived from the UniHI database.

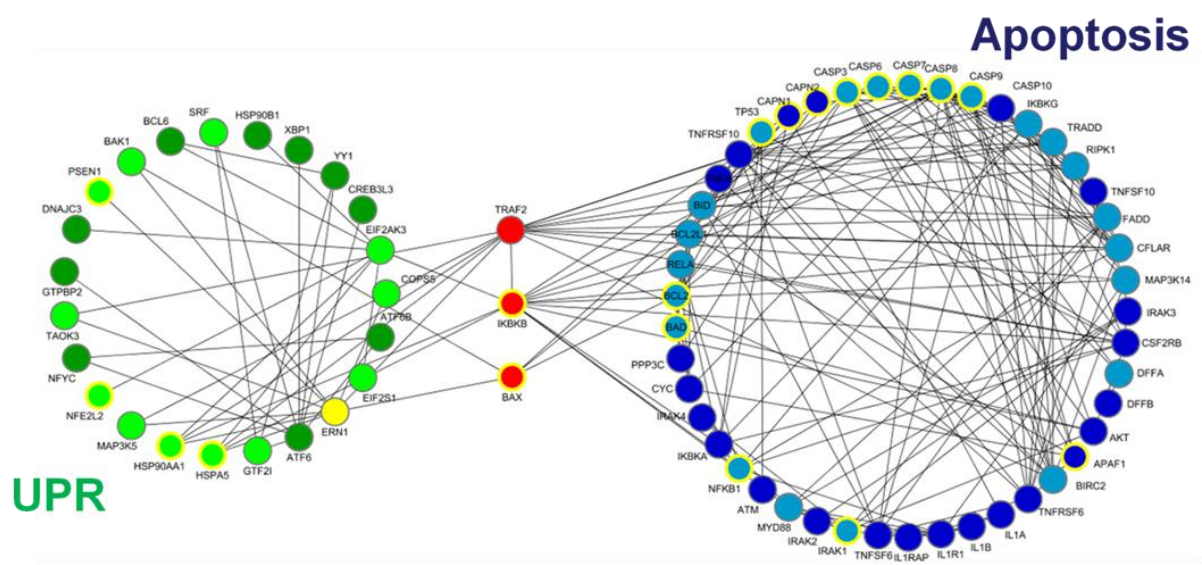

Figure 5: Network representation of core UPR and apoptosis. UPR proteins with interactions to apoptosis were shaded by dark green, apoptosis protein with interactions to UPR by dark purple. Interconnecting proteins are shown in blue. HD associated genes are highlighted by yellow circles. 


\section{Neuroinflammation in HD}

Inflammation is a immunological process usually carried out by the blood vascular system to counteract disease, and to fight foreign antigens against invasion. Within the brain, microglia and astrocytes play important immunological functions. Until very recently little was known about inflammatory molecules in HD.

Recent studies, however, revealed a distinct profile of inflammatory mediators from post-mortem human HD tissue [6,7]. Important in this context are following observations:

- In general, activated microglia exert their diverse effects on neurons and macroglia (astrocytes and oligodendrocytes) through the release of cytoprotective agents such as growth factors, plasminogen, and neuroprotective cytokine as well as cytotoxic substances such as oxygen radicals, nitric oxide, glutamate, proteases, and neurotoxic cytokines. Notably, one of the earliest reports describing microglial abnormalities in HD was by Singhrao et al [9]. Inflammatory mediators such as IL-1 $\beta$ and TNF- $\alpha$ were increased only in the striatum, whereas IL-6, IL-8 and MMP-9 were also upregulated in cortex and in the cerebellum [7].

- Microglial cell counts were considerably increased in the caudate putamen of HD and these microglial cells expressed increased amounts of complement factors [9]. A more detailed investigation of microglial morphological changes associated with HD was performed by Sapp et al. [14]. The authors localized morphologically activated microglial cells in the neostriatum, cortex and globus palidus as well as in adjoining white matter of HD brains. Additionally, PET studies using the ligand for benzodiazepine receptor (PK-11195), which labels activated microglia have been employed to study of neuroinflammation. Using this technique, Tai et al $[15,16]$ demonstrated that microglial activation in HD patients correlates with disease progression as assessed by loss of dopamine D2 receptor binding sites. Interestingly Tai et al. could also demonstrate that microglial activation and release of cytokine IL6 is observed in presymptomatic HD gene carriers and can be detected up to 15 years before predicted age of onset. These findings indicate the microglial inflammatory activation is an early event associated with subclinical progression of $\mathrm{HD}$ and may constitute a target for early therapeutic intervention.

- Earlier studies performed by one of us in Alzheimer disease observed strong correlations of redox alterations to inflammatory stress responses [17,18]. Here, we demonstrated an intermediary signaling role of ceramide in activation of stress kinase cascades linking oxidative stress with inflammatory induction of enzymes iNOS, COX-2 with the release of NO, and pro-inflammatory cytokines TNF- $\alpha$, IL-
$1 \beta$ and IL-6. Inhibition of ceramide generation and or pro-oxidant responses by use of ceramide synthesis blocker (such as fumonisin B1, sphingomyelinase inhibitors 3-o-methyl sphingomyelin), and anti-oxidant Vitamin E, completely prevent these proinflammatory stress responses. In this context, AICAR, an AMP kinase activator, has demonstrated has a therapeutic potential in curbing proinflammatory responses as well preventing neuronal cell apoptosis [18].

\section{Possible experimental follow-up}

Based on the undertaken review, we hypothesize that $\mathrm{mHtt}$ generated ER stress might lead to apoptotic cascades in neuronal stress and might trigger inflammatory stress responses in adjacent glial cells in the diasease pathogenesis. A first experimental analysis in primary glial cells could elucidate the inflammatory stress in HD pathology. Initially, it is important to examine the role of $\mathrm{mHtt}$ in microglia cells i.e. to determine whether $\mathrm{mHtt}$ leads to ER stress in microglia. In case of UPR activation, the LPS/Smase dependent cytokine and chemokine release can be measured to verify inflammatory signaling. An interesting sideline would be whether occurrence of ER stress might be correlated with mitochondrial stress $[19,20]$. Hence, it is would be relevant to demonstrate possible alterations in mitochondrial protein expression and measurement of redox indicators (reduced glutathione, generation of reactive oxygen species as well expression of redox regulating enzymes such as superoxide dismutases $(\mathrm{Cu} / \mathrm{Zn}$ and $\mathrm{Mn}-\mathrm{SOD}$, catalase). Finally, the role of excitotoxic glutamate release mechanisms in neuronal cell death and apoptosis is of relevance here, as glial cell dysfunction and associated inflammatory stress responses might cause imbalances in glutamate/calcium homeostasis [21]. Figure 6 presents an initial working model for the experimental study of ER stress in glial cells.

\section{Acknowledgements}

The presented work was supported by the CHDI Foundation (http://www.chdifoundation.org/). 


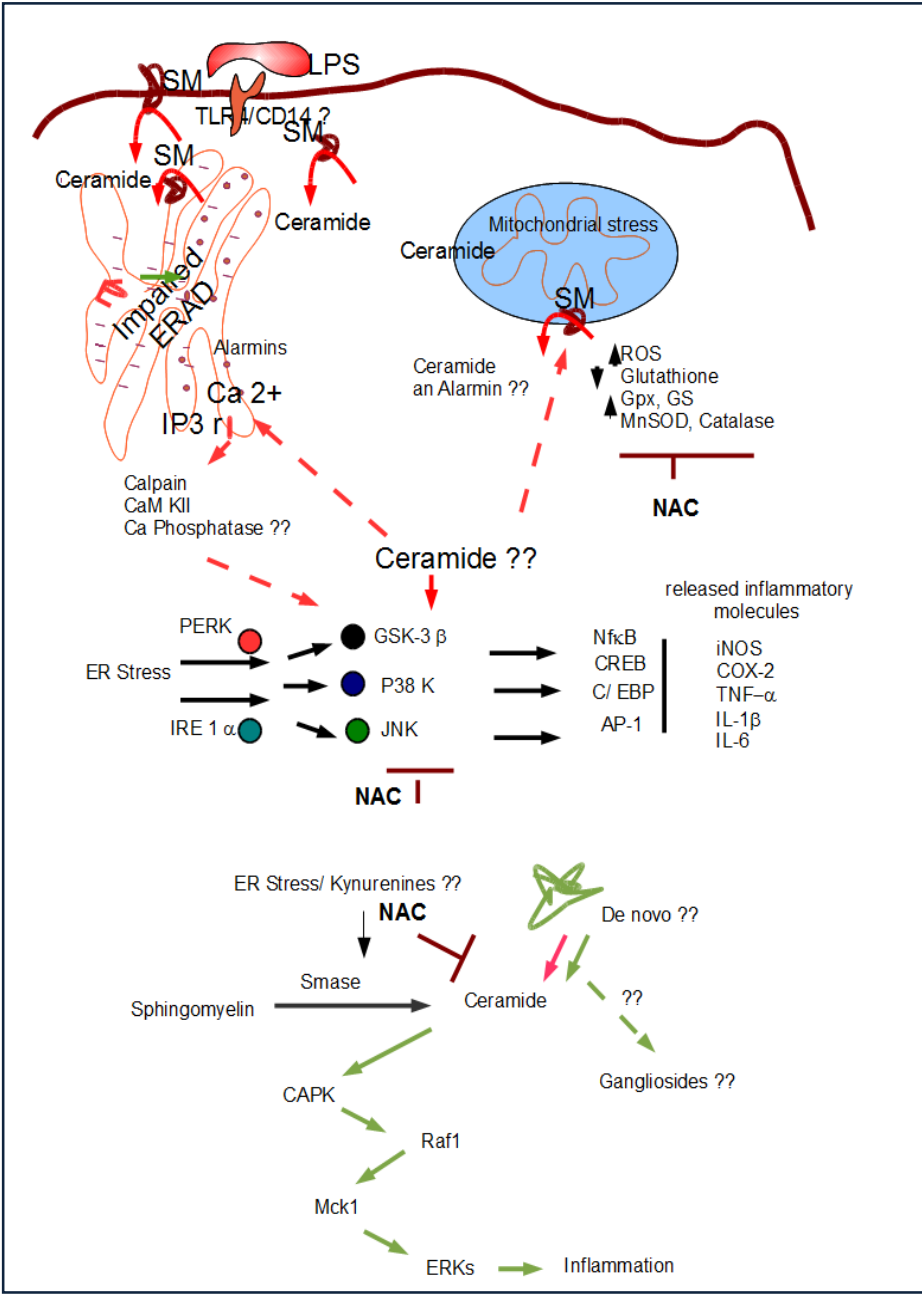

Figure 6. Endoplasmic stress (ER) cascade embedded in inflammatory stress signaling network in astroglial cells. Binding by LPS to TLR-4 (CD14) receptor can experimentally be used to trigger a downstream Smase/Cer signaling and to activate various stress kinases leading further to activation of several transcription factors and thus inducing the release of various inflammatory molecules. ER stress can be induced by inhibiting of ERAD and the effects on inflammatory signaling can be studied. Ceramide, the intermediary messenger generated as a result of ER stress, likely activates the stress signaling. The red dotted lines represent pathways which are yet unclear. N-acetyl cysteins (NAC) a known anti-oxidant as well an inflammatory blocker might prove beneficial in curbing these responses as well for possible therapeutics.

\section{References}

1. The Huntington's Disease Collaborative Research Group. A novel gene containing a trinucleotide repeat that is expanded and unstable on Huntington's disease chromosomes. Cell 1993, 72:971-983.

2. Rutkowski DT and Kaufman RJ, That which does not kill me makes me stronger: adapting to chronic ER stress.. Trends Biochem Science 2007 32(10):469-76.

3. Duennwald ML and Linquist S. Impaired ERAD and ER stress are early and specific events in polyglutamine toxicity. Genes Dev. 2008 1;22(23):3308-19

4. Matus S, Lisbona F, Torres M, León C, Thielen P, Hetz C. The stress rheostat: an interplay between the unfolded protein response (UPR) and autophagy in neurodegeneration. Curr Mol Med. 2008 8(3):15772

5. Zhang K, Kaufman RJ. From endoplasmicreticulum stress to the inflammatory response. Nature. 2008 24;454(7203):455-62.
6. Möller T. Neuroinflammation in Huntington's disease. J Neural Transm. 2010 117(8):1001-87. Silvestroni A, Faull RL, Strand AD, Moller Distinct neuroinflammatory profile in post-mortem human Huntington's disease. T (2009) Neuroreport 20:1098-1103

8. Dalrymple A, Wild EJ, Joubert R, Sathasivam K, Björkqvist M, Petersén A, Jackson GS, Isaacs JD, $\underline{\text { Kristiansen M, Bates GP, Leavitt BR, Keir G, Ward }}$ $\underline{M}$, Tabrizi SJ. Proteomic profiling of plasma in Huntington's disease reveals neuroinflammatory activation and biomarker candidates. $\underline{\text { Proteome }}$ Res. 2007 6(7):2833-40.

9. Singhrao SK, Neal JW, Morgan BP, Gasque P. Increased complement biosynthesis by microglia and complement activation on neurons in Huntington's disease. Exp Neurol 1999 159:362- 376.

10. Kuhn A, Goldstein DR, Hodges A, Strand AD, Sengstag T, Kooperberg C, Becanovic K, Pouladi MA, Sathasivam K, Cha JH, et al: Mutant huntingtin's effects on striatal gene expression in mice recapitulate changes observed in human 
Huntington's disease brain and do not differ with mutant huntingtin length or wild-type huntingtin dosage. Hum Mol Genet 2007, 16:1845-1861.

11. Hodges A, Hughes G, Brooks S, Elliston L, Holmans P, Dunnett SB, Jones L., Brain gene expression correlates with changes in behavior in the R6/1 mouse model of Huntington's disease. Genes Brain Behav. 2008 7(3):288-99.

12. Hodges A, Strand AD, Aragaki AK, Kuhn A, Sengstag T, Hughes G, Elliston LA, Hartog C, Goldstein DR, Thu D, et al: Regional and cellular gene expression changes in human Huntington's disease brain. Hum Mol Genet 2006, 15:965-977.

13. Nishitoh H, Matsuzawa A, Tobiume K, Saegusa K, Takeda K, Inoue K, Hori S, Kakizuka A, Ichijo H., ASK1 is essential for endoplasmic reticulum stress-induced neuronal cell death triggered by expanded polyglutamine repeats. Genes Dev. 2002 16(11):1345-55.

14. Sapp E, Kegel KB, Aronin N, Hashikawa T, Uchiyama Y, Tohyama K, Bhide PG, Vonsattel JP, DiFiglia M Early and progressive accumulation of reactive microglia in the Huntington disease brain..J Neuropathol Exp Neurol 2001.60:161-172.

15. Tai YF, Pavese N, Gerhard A, Tabrizi SJ, Barker RA, Brooks DJ, Piccini P Microglial activation in presymptomatic Huntington's disease gene carriers. Brain (2007) 130:1759-1766.
16. Tai YF, Pavese N, Gerhard A, Tabrizi SJ, Barker RA, Brooks DJ,Piccini P Microglial activation correlates with severity in Huntington disease: a clinical and PET study. Neurology. 2006. 66(11):1638-43.

17. Inflammatory Ayasolla K, Khan M, Singh AK, and Singh I. Mediator and beta-amyloid (25-35)induced ceramide generation and iNOS expression are inhibited by vitamin E. Free Radic Biol Med. 2004;37(3):325-38

18. Ayasolla KR, Singh AK, Singh I.J. 5aminoimidazole-4-carboxamide-1-beta-4-

ribofuranoside (AICAR) attenuates the expression of LPS- and Abeta peptide-induced inflammatory mediators in astroglia. Neuroinflammation. 2002:21

19. Cui L, Jeong H, Borovecki F, Parkhurst CN, Tanese N, Krainc D. Transcriptional repression of PGC-1alpha by mutant huntingtin leads to mitochondrial dysfunction and neurodegeneration. Cell. 2006 127(1):59-69.

20. Quintanilla RA, Johnson GV. Role of mitochondrial dysfunction in the pathogenesis of Huntington's disease. Brain Res Bull. 2009;80(45):242-7.

21.Levine MS, Cepeda C, André VM. Location, location, location: contrasting roles of synaptic and extrasynaptic NMDA receptors in Huntington's disease.. Neuron. 2010 65(2):145-7. 